\title{
SAV Method Applied to Fractional Allen-Cahn Equation
}

\author{
Xiaolan Zhou, Mejdi Azaiez, and Chuanju Xu
}

\section{Introduction}

The Allen-Cahn equation was originally introduced to describe the motion of antiphase boundaries in crystalline solids [1]. There have been a large body of work on numerical analysis of Allen-Cahn equations (cf. [2-5] and the references therein). We aim in this paper to use the SAV scheme, recently introduced and analyzed by a number of researchers; see, e.g., [5] and the references therein, to approximate the solution of the fractional version of the Allen-Cahn model. It consists in finding $\phi: \Omega \times(0, T] \rightarrow \mathbb{R}$ solution of

$$
\left\{\begin{array}{l}
\frac{\partial \phi}{\partial t}+\gamma\left((-\Delta)^{s} \phi+f(\phi)\right)=0, \quad \forall(\boldsymbol{x}, t) \in \Omega \times(0, T], \\
\left.\nabla \phi \cdot \boldsymbol{n}\right|_{\partial \Omega}=0, \quad \forall t \in(0, T] \\
\phi(t=0)=\phi_{0}(\boldsymbol{x}), \quad \forall \boldsymbol{x} \in \Omega .
\end{array}\right.
$$

In the above, $\gamma$ is a positive kinetic coefficient, $s \in(0,1), \Omega \subset \mathbb{R}^{d}$ is a bounded domain, $\boldsymbol{n}$ is the outward normal, $f(\phi)=F^{\prime}(\phi)$ with a given function $F(\phi)=$ $\frac{1}{4 \varepsilon^{2}}\left(\phi^{2}-1\right)^{2}$ being the Ginzburg-Landau double-well potential. The phase field $\phi$

X. Zhou $\cdot$ C. Xu $(\bowtie)$

School of Mathematical Sciences, Xiamen University, Xiamen, China

e-mail: xlzhou@stu.xmu.edu.cn; cjxu@xmu.edu.cn

M. Azaiez

School of Mathematical Sciences, Xiamen University, Xiamen, China

Bordeaux INP, I2M (UMR CNRS 5295), Université de Bordeaux, Pessac, France

e-mail: azaiez@enscbp.fr 
is such that

$$
\phi= \begin{cases}1, & \text { phase } 1 \\ -1, & \text { phase } 2\end{cases}
$$

and $\varepsilon$ represents the thickness of the smooth transition layer connecting the two phases, which is small compared to the characteristic length of the system scale. The homogeneous Neumann boundary condition implies that no mass loss occurs across the boundary walls.

Among the different definitions of fractional Laplacians (see [6, 7] for a quantitative assessment of new numerical methods as well as available state-of-theart methods for discretizing the fractional Laplacians problems), we choose in this paper to focus on the fractional spectral definition. It is defined by

$$
(-\Delta)^{s} u:=\sum_{i \in \mathbb{N}} a_{i} \lambda_{i}^{s} e_{i}
$$

where $\lambda_{i}, e_{i}$ are the eigenvalues and eigenfunctions of the Laplace operator $-\Delta$ in $\Omega$ with homogeneous Neumann boundary condition, i.e., they satisfy

$$
\left\{\begin{array}{l}
-\Delta e_{i}=\lambda_{i} e_{i}, \quad x \in \Omega \\
\left.\nabla e_{i} \cdot \boldsymbol{n}\right|_{\partial \Omega}=0
\end{array}\right.
$$

While, $a_{i}$ represents the projection of $u$ on the direction $e_{i}, a_{i}=\left(u, e_{i}\right)_{L_{\Omega}^{2}}$. The spectral fractional Laplacian is nonlocal on the interior for noninteger $s \in(0,1)$. We see that to compute the inner product $a_{i}=\left(u, e_{i}\right)_{L_{\Omega}^{2}}$, it suffices for $u$ to be defined on the interior of $\Omega$. No information about $u$ on the exterior $R^{d} \backslash \Omega$ is required. Thus, from a conceptual viewpoint, in boundary value problems the spectral fractional Laplacian can admit the same type of boundary conditions as the standard, local Laplacian $-\Delta$. In this paper, we let $\Omega=]-1,1\left[^{2}\right.$. Set $u(\boldsymbol{x}, t)=$ $\sum_{n=1}^{\infty} \sum_{m=1}^{\infty} a_{m, n}(t) e_{m, n}(\boldsymbol{x})$, where $e_{m, n}$ are the orthogonal eigenfunctions of the Laplace operator with homogeneous Neumann boundary conditions and $\lambda_{m, n}$ are the corresponding eigenvalues. Then we define the spectral fractional Laplacian as,

$$
(-\Delta)^{s} u(\boldsymbol{x}, t):=\sum_{n=1}^{\infty} \sum_{m=1}^{\infty} \lambda_{m, n}^{s} a_{m, n}(t) e_{m, n}(\boldsymbol{x}), \quad 0<s<1, \forall u \in H^{s}(\Omega) .
$$


Here

$H^{s}(\Omega):=\left\{u=\sum_{n=1}^{\infty} \sum_{m=1}^{\infty} a_{m, n} e_{m, n} \in L^{2}(\Omega):|u|_{s}:=\left(\sum_{n=1}^{\infty} \sum_{m=1}^{\infty} \lambda_{m, n}^{s} a_{m, n}^{2}\right)^{1 / 2}<\infty\right\}$.

The rest of this paper in organized as follows. In Sect. 2, we present briefly the spectral method by giving some notations and reminders. The fractional Laplace operator and its possible applications is discussed in Sect. 3. To demonstrate the applicability of the approximative fractional Laplacian for real applications, we consider a fractional Allen-Cahn equation (FACE). Based on the scalar auxiliary variable (SAV) approach, we construct an unconditionally second-order energy stable BDF scheme (SAV/BDF2) for FACE. We present numerical results for a test case as well as a benchmark example in Sect. 4.

\section{Spatial Discretizations}

We limit here the description of the spectral approximation to the introduction of some notations and reminders (see [8,9]). For complex domain, we can use spectral element method [10]. Let $\Sigma=\left\{\left(\xi_{i}, \rho_{i}\right) ; 0 \leq i \leq N\right\}$ denote the sets of Gauss-Lobatto-Legendre quadrature nodes and weights associated to polynomials of degree $N$. These quantities are such that on $\Lambda:=]-1,+1[$

$$
\forall \phi \in \mathbb{P}_{2 N-1}(\Lambda), \quad \int_{-1}^{+1} \phi(\xi) d \xi=\sum_{j=0}^{N} \phi\left(\xi_{j}\right) \rho_{j}
$$

where $\mathbb{P}_{N}(\Lambda)$ denotes the space of polynomials of degree $\leq N$. We recall that the nodes $\xi_{i}(0 \leq i \leq N)$ are solution to $\left(1-x^{2}\right) L_{N}^{\prime}(x)=0$, where $L_{N}$ denotes the Legendre polynomial of degree $N$.

The canonical polynomial interpolation basis $h_{i}(x) \in \mathbb{P}_{N}(\Lambda)$ built on $\Sigma$ is given by the relationships:

$$
h_{i}(x)=-\frac{1}{N(N+1)} \frac{1}{L_{N}\left(\xi_{i}\right)} \frac{\left(1-x^{2}\right) L_{N}^{\prime}(x)}{\left(x-\xi_{i}\right)}, \quad-1 \leq x \leq+1, \quad 0 \leq i \leq N,
$$

with the elementary cardinality property

$$
h_{i}\left(\xi_{j}\right)=\delta_{i j}, \quad 0 \leq i, j \leq N,
$$

where $\delta_{i j}$ is Kronecker's delta symbol. 
In the sequel the phase field $\phi$ will be approximated in space variable by suitable polynomial functions $\phi_{N}$ as follows

$$
\phi_{N}(\boldsymbol{x}, t)=\sum_{i=0}^{N} \sum_{j=0}^{N} \alpha_{i, j}(t) h_{i}(x) h_{j}(y) .
$$

The $L^{2}$-inner products involved in the calculation will be achieved using GaussLobatto-Legendre quadrature, which reads: for all continuous functions $\varphi$ and $\psi$ in $\bar{\Omega}$,

$$
(\varphi, \psi) \approx(\varphi, \psi)_{N}:=\sum_{i=0}^{N} \sum_{j=0}^{N} \varphi\left(\xi_{i}\right) \psi\left(\xi_{j}\right) \rho_{i} \rho_{j}
$$

\section{Scalar Auxiliary Variable (SAV) Approach for FACE}

SAV approach was introduced in $[4,5]$ to solve gradient flows. The main purpose of this section is to construct efficient unconditionally stable scheme based on this approach for (1.1).

Throughout the paper, we assume there exists a constant $C_{0}$ such that $\int_{\Omega} F(\phi) \mathrm{d} \boldsymbol{x}+C_{0}>0$. We first introduce a scalar auxiliary variable

$$
r(t):=\sqrt{\int_{\Omega} F(\phi) \mathrm{d} x+C_{0}} .
$$

Then, we rewrite the phase-field equation (1.1) under an equivalent form as: find $\phi:(0, T] \times \Omega \rightarrow \mathbb{R}$ and $r:(0, T] \rightarrow \mathbb{R}$, such that

$$
\left\{\begin{aligned}
\frac{\partial \phi}{\partial t} & =\gamma \mu,\left.\quad \nabla \phi \cdot \boldsymbol{n}\right|_{\partial \Omega}=0 \\
\mu & =-(-\Delta)^{s} \phi-\frac{r(t)}{\sqrt{\int_{\Omega} F(\phi) d \boldsymbol{x}+C_{1}}} f(\phi) \\
\frac{\mathrm{d} r}{\mathrm{~d} t} & =\frac{1}{2 \sqrt{\int_{\Omega} F(\phi) d \boldsymbol{x}+C_{1}}} \int_{\Omega} f(\phi) \frac{\partial \phi}{\partial t} d \boldsymbol{x}
\end{aligned}\right.
$$

Theorem 3.1 If $\phi \in L^{2}\left((0, T], H^{s}(\Omega)\right), 0<s<1$, is the solution of equations (3.1), then we have the following energy dissipation law

$$
\frac{d}{d t}\left(r^{2}+\frac{1}{2}|\phi|_{s}^{2}\right)=-\gamma\|\mu\|_{0}^{2} .
$$


Proof Taking the inner product of the first two equations with $\mu, \frac{\partial \phi}{\partial t}$ respectively, and multiplying the third equation with $2 r(t)$, then adding them together, We obtain

$$
-\gamma\|\mu\|_{0}^{2}=\frac{d}{d t}\left(r^{2}\right)+\left((-\Delta)^{s} \phi, \phi_{t}\right) .
$$

Let $\phi(\boldsymbol{x}, t)=\sum_{m, n=1}^{\infty} a_{m, n}(t) e_{m, n}(\boldsymbol{x})$ and taking advantage of the orthogonality of $\left\{e_{m, n}\right\}$, we verify

$$
\left((-\Delta)^{s} \phi, \phi_{t}\right)=\sum_{m, n=1}^{\infty} \lambda_{m, n}^{s} a_{m, n}(t) a_{m, n}^{\prime}(t)=\frac{1}{2} \frac{d}{d t}\left(\sum_{m, n=1}^{\infty} \lambda_{m, n}^{s} a_{m, n}^{2}(t)\right)=\frac{1}{2} \frac{d}{d t}|\phi|_{s}^{2} .
$$

Then combining (3.3) and (3.4) proves (3.2).

The energy law (3.2) means that the SAV approach (3.1) makes the modified energy

$$
H(\phi)=r^{2}+\frac{1}{2}|\phi|_{s}^{2}
$$

decay in time.

Now we construct a second-order semi-implicit scheme for the system (3.1). Given initial conditions $\phi^{0}=\phi_{0}$, and let $r^{0}=\sqrt{\int_{\Omega} F\left(\phi^{0}\right) d \boldsymbol{x}+C_{0}}$, find $\phi^{n+1} \in$ $H^{s}(\Omega)$ and $r^{n+1} \in \mathbb{R}, n=1, \ldots$, such that

$$
\begin{aligned}
\frac{3 \phi^{n+1}-4 \phi^{n}+\phi^{n-1}}{2 \Delta t} & =\gamma \mu^{n+1} \\
\mu^{n+1} & =-(-\Delta)^{s} \phi^{n+1}-\frac{r^{n+1}}{\sqrt{\int_{\Omega} F\left(\bar{\phi}^{n+1}\right) d x+C_{0}} f\left(\bar{\phi}^{n+1}\right)} \\
\frac{3 r^{n+1}-4 r^{n}+r^{n-1}}{2 \Delta t} & =\frac{1}{2 \sqrt{\int_{\Omega} F\left(\bar{\phi}^{n+1}\right) d x+C_{0}}} \int_{\Omega} f\left(\bar{\phi}^{n+1}\right) \frac{3 \phi^{n+1}-4 \phi^{n}+\phi^{n-1}}{2 \Delta t} d \boldsymbol{x}
\end{aligned}
$$

In the above, $\bar{\phi}^{n+1}$ can be any explicit approximation of $\phi\left(t^{n+1}\right)$ with an error of $\mathcal{O}\left(\Delta t^{2}\right)$. For instance, we may choose the following one

$$
\bar{\phi}^{n+1}=2 \phi^{n}-\phi^{n-1} .
$$


Theorem 3.2 The scheme (3.5)-(3.7) is unconditionally stable in the sense that

$$
\frac{1}{\Delta t}\left(\tilde{H}\left[\left(\phi^{n+1}, r^{n+1}\right),\left(\phi^{n}, r^{n}\right)\right]-\tilde{H}\left[\left(\phi^{n}, r^{n}\right),\left(\phi^{n-1}, r^{n-1}\right)\right]\right) \leq-\gamma\left\|\mu^{n+1}\right\|_{0}^{2},
$$

with the modified energy

$$
\tilde{H}\left[\left(\phi^{n+1}, r^{n+1}\right),\left(\phi^{n}, r^{n}\right)\right]=\frac{1}{4}\left(\left|\phi^{n+1}\right|_{s}^{2}+\left|2 \phi^{n+1}-\phi^{n}\right|_{s}^{2}\right)+\frac{1}{2}\left(\left(r^{n+1}\right)^{2}+\left(2 r^{n+1}-r^{n}\right)^{2}\right) .
$$

Proof The result can be directly deduced from taking the inner product of the first two equations (3.5) and (3.6) with $\mu^{n+1}$ and $\frac{3 \phi^{n+1}-4 \phi^{n}+\phi^{n-1}}{2 \Delta t}$ respectively, and multiplying the third equation (3.7) with $2 r^{n+1}$, then using the following identity:

$$
\begin{aligned}
2\left(a^{k+1}, 3 a^{k+1}-4 a^{k}+a^{k-1}\right)= & \left\|a^{k+1}\right\|^{2}+\left\|2 a^{k+1}-a^{k}\right\|^{2}+\left\|a^{k+1}-2 a^{k}+a^{k-1}\right\|^{2} \\
& -\left\|a^{k}\right\|^{2}-\left\|2 a^{k}-a^{k-1}\right\|^{2} .
\end{aligned}
$$

\subsection{Implementation}

Besides its unconditional stability, a most remarkable feature of the above scheme is that it can be solved very efficiently. Indeed, by inserting (3.6) and (3.7) into (3.5), and let $\overline{\mathcal{F}}^{n+1}:=\int_{\Omega} F\left(\bar{\phi}^{n+1}\right) d \boldsymbol{x}+C_{0}$, we obtain

$$
\left(\frac{3}{2 \gamma \Delta t} I+(-\Delta)^{s}\right) \phi^{n+1}+\frac{\left(f\left(\bar{\phi}^{n+1}\right), \phi^{n+1}\right)}{2 \overline{\mathcal{F}}^{n+1}} f\left(\bar{\phi}^{n+1}\right)=g^{n},
$$

where

$$
g^{n}:=\frac{4 \phi^{n}-\phi^{n-1}}{2 \gamma \Delta t}-\left(\frac{4 r^{n}-r^{n-1}}{3 \sqrt{\overline{\mathcal{F}}^{n+1}}}-\frac{\left(f\left(\bar{\phi}^{n+1}\right), \frac{4 \phi^{n}+\phi^{n-1}}{3}\right)}{2 \overline{\mathcal{F}}^{n+1}}\right) f\left(\bar{\phi}^{n+1}\right) .
$$

We shall first determine $\left(f\left(\bar{\phi}^{n+1}\right), \phi^{n+1}\right)$ from (3.10). To this end we multiply (3.10) by $\left(\frac{3}{2 \gamma \Delta t} I+(-\Delta)^{s}\right)^{-1}$ and take the inner product by $f\left(\bar{\phi}^{n+1}\right)$ to get

$$
\left(f\left(\bar{\phi}^{n+1}\right), \phi^{n+1}\right)+\frac{\left(f\left(\bar{\phi}^{n+1}\right), \phi^{n+1}\right)}{2 \overline{\mathcal{F}}^{n+1}}\left(f\left(\bar{\phi}^{n+1}\right), \beta^{n+1}\right)=\left(f\left(\bar{\phi}^{n+1}\right), \alpha^{n+1}\right),
$$


with

$$
\alpha^{n+1}=\left(\frac{3}{2 \gamma \Delta t} I+(-\Delta)^{s}\right)^{-1} g^{n}, \quad \beta^{n+1}=\left(\frac{3}{2 \gamma \Delta t} I+(-\Delta)^{s}\right)^{-1} f\left(\bar{\phi}^{n+1}\right) .
$$

Then, we have

$$
\left(f\left(\bar{\phi}^{n+1}\right), \phi^{n+1}\right)=\frac{\left(f\left(\bar{\phi}^{n+1}\right), \alpha^{n+1}\right)}{1+\frac{1}{2 \overline{\mathcal{F}}^{n+1}}\left(f\left(\bar{\phi}^{n+1}\right), \beta^{n+1}\right)} .
$$

Thus we obtain an expression to compute $\phi^{n+1}$ by bringing back (3.14) into (3.10):

$$
\phi^{n+1}=\alpha^{n+1}-\frac{\left(f\left(\bar{\phi}^{n+1}\right), \alpha^{n+1}\right)}{2 \overline{\mathcal{F}}^{n+1}+\left(f\left(\bar{\phi}^{n+1}\right), \beta^{n+1}\right)} \beta^{n+1} .
$$

Finally we compute $r^{n+1}$ through

$$
r^{n+1}=\frac{4 r^{n}-r^{n-1}}{3}+\frac{\left(f\left(\bar{\phi}^{n+1}\right), \phi^{n+1}\right)-\left(f\left(\bar{\phi}^{n+1}\right), \frac{4 \phi^{n}-\phi^{n-1}}{3}\right)}{2 \sqrt{\overline{\mathcal{F}}^{n+1}}} .
$$

We now summarize the algorithm of the Scalar Auxiliary Variable approach/SemiImplicit Second-Order Scheme (3.5)-(3.7) as follows:

1. Set $\bar{\phi}^{n+1}=2 \phi^{n}-\phi^{n-1}, \quad \overline{\mathcal{F}}^{n+1}=\int_{\Omega} F\left(\bar{\phi}^{n+1}\right) d \boldsymbol{x}+C_{0}$,

$$
\begin{aligned}
\tilde{c}_{0} & =\left(f\left(\bar{\phi}^{n+1}\right), 4 \phi^{n}-\phi^{n-1}\right) / 3, \quad \tilde{c}_{1}=\frac{4 r^{n}-r^{n-1}}{3 \sqrt{\overline{\mathcal{F}}^{n+1}}}-\frac{\tilde{c}_{0}}{2 \overline{\mathcal{F}}^{n+1}}, \\
g^{n} & =\frac{4 \phi^{n}-\phi^{n-1}}{2 \gamma \Delta t}-\tilde{c}_{1} f\left(\bar{\phi}^{n+1}\right) ;
\end{aligned}
$$

2. Solve $\frac{3}{2 \gamma \Delta t} \beta^{n+1}+(-\Delta)^{s} \beta^{n+1}=f\left(\bar{\phi}^{n+1}\right)$;

3. Solve $\frac{3}{2 \gamma \Delta t} \alpha^{n+1}+(-\Delta)^{s} \alpha^{n+1}=g^{n}$;

4. Compute $\tilde{c}_{2}=\left(f\left(\bar{\phi}^{n+1}\right), \beta^{n+1}\right), \quad \tilde{c}_{3}=\left(f\left(\bar{\phi}^{n+1}\right), \alpha^{n+1}\right), \quad \tilde{c}_{4}=$ $\tilde{c}_{3} /\left(2 \overline{\mathcal{F}}^{n+1}+\tilde{c}_{2}\right)$

5. Compute $\phi^{n+1}=\alpha^{n+1}-\tilde{c}_{4} \beta^{n+1}, r^{n+1}=\frac{4 r^{n}-r^{n-1}}{3}+$

$$
\frac{\left(f\left(\bar{\phi}^{n+1}\right), \phi^{n+1}\right)-\tilde{c}_{0}}{2 \sqrt{\overline{\mathcal{F}}^{n+1}}} .
$$




\section{Numerical Results and Discussion}

In this section, we first present a numerical example to illustrate the efficiency of the SAV scheme in terms of stability and accuracy. We then use the proposed scheme to simulate a benchmark problem.

\subsection{Test of the Convergence Order}

In order to validate the proposed SAV/BDF2 scheme for the fractional phase-field equation, we consider a fabricated forcing term so that the exact solution to (1.1) is $\phi(\boldsymbol{x}, t)=\sin (t) \cos (\pi x) \cos (\pi y)$. In this test we set $\gamma=1, \Omega=]-1,1\left[^{2}\right.$, and the nonlinear term is given by $f(\phi)=\phi\left(\phi^{2}-1\right)$.

In the calculation we use polynomial degree $32 \times 32$ for the spatial discretization, which is large enough so that the spatial discretization error is negligible compared to the temporal error. Figure 1 shows the $L^{2}$-errors at $T=1.0$ in $\log$-log scale as a function of the time step size for several fractional orders. It is observed from this figure that the convergence rate of the time stepping scheme is exactly second order as expected for all tested values of $s$. It is worthy to mention that no numerical instability was observed for all time step sizes used in the calculation. This implies that the proposed scheme is unconditionally stable.

Fig. $1 L^{2}$-errors at $T=1.0$ in $\log$-log scale with respect to the time step size $\Delta t$ for different fractional order $s$

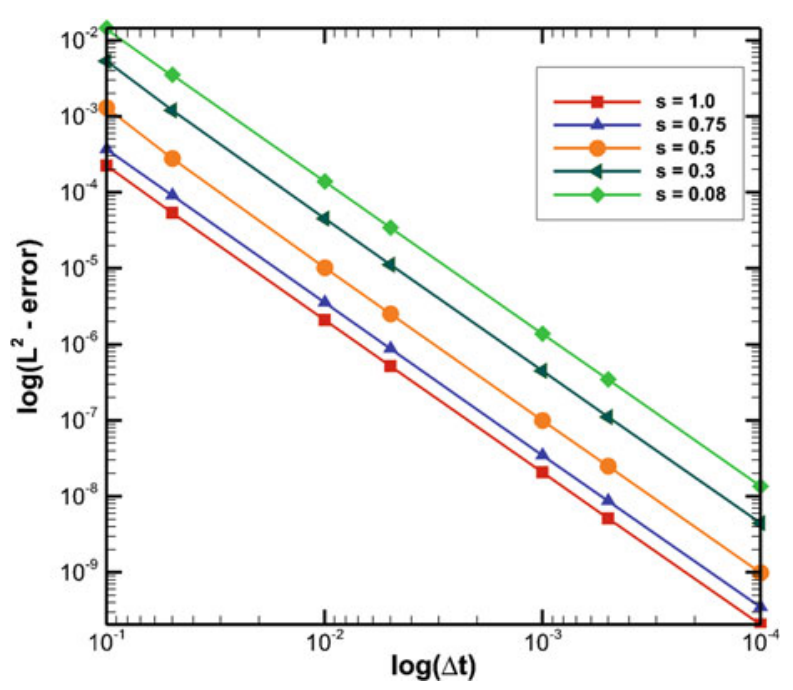




\subsection{Benchmark Test}

In this subsection, we apply the SAV/BDF2 scheme to the fractional version of a classical benchmark problem (cf. [11]) that we describe below. Our main purpose in this test is to demonstrate the applicability of the constructed method for the FACE. We are particularly interested in numerically investigating the impact of the fractional order on the evolution of the phase interface.

At the initial state, there is a circular phase interface of the radius $R_{0}=100$ in the rectangular domain $]-128,128\left[^{2}\right.$. In other words, the initial condition is given by

$$
\phi(x, 0)=\left\{\begin{array}{lc}
1, & |x|^{2}<100^{2} \\
-1, & |x|^{2} \geq 100^{2}
\end{array}\right.
$$

Such a circular interface is unstable and the driving force will make it shrink and eventually disappear. It has been shown that in the limit that the radius of the circle is much larger than the interfacial thickness, the velocity and the radius of the moving interface are given (see [1]) by

$$
V=\frac{d R}{d t}=-\frac{1}{R}, \quad R(t)=\sqrt{R_{0}^{2}-2 t} .
$$

In the implementation we map the computational domain $]-128,128\left[^{2}\right.$ to $]-$ $1,1\left[^{2}\right.$. Therefore actually we are led to solve the fractional Allen-Cahn equation (1.1) with the coefficients $\gamma=1 / 128^{2}$ and $\varepsilon=0.0078$. In the simulation, the space resolution is set to $N=512$, and the time step size is $\Delta t=0.1$. The computed radius $R(t)$ for $s=1$ using the SAV/BDF2 scheme is plotted in Fig. 2. We observe that $R(t)$ keeps monotonously decreasing and very close to the sharp interface limit value. This confirms the accuracy of the proposed method, at least in the case $s=1$.

Next we apply the proposed scheme to investigate the impact of the fractional order on the radius behavior. In Fig. 3 we present the numerical radius evolution for a number of the fractional orders. Specifically, Fig. 4 shows the circle shrinking for fractional orders $s=1.0,0.9,0.8$. It is clearly indicated that the radius decay rate slow down when the fractional order decreases. However, for the time being the physical meaning and mathematical explanation of this phenomena remain unknown. We plan to address this issue in future work. 


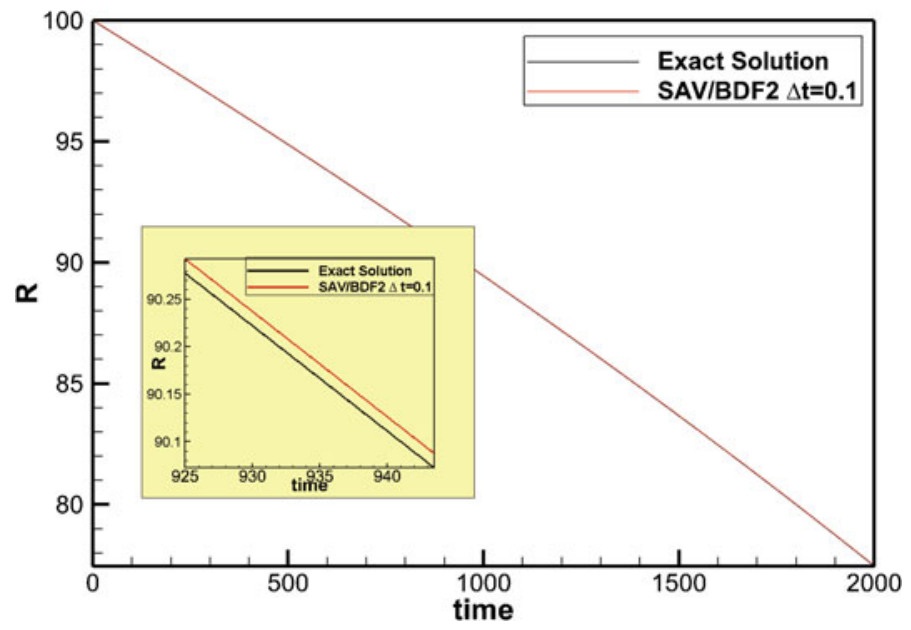

Fig. 2 The evolution of radius $R(t)$ : comparison of the exact solution and numerical result in the case $s=1$

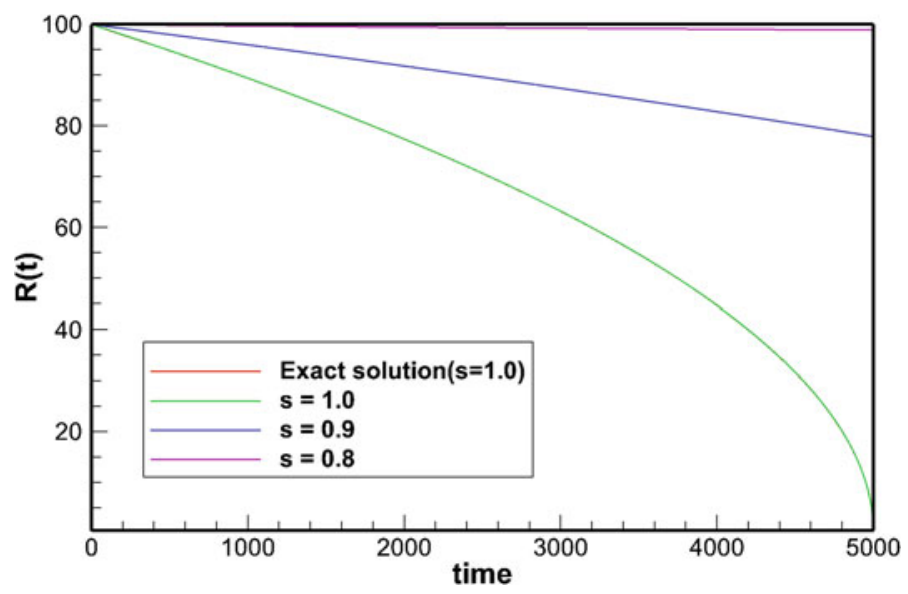

Fig. 3 Evolution of the radius for different fractional order $s$ : impact of the order on the radius decay rate 

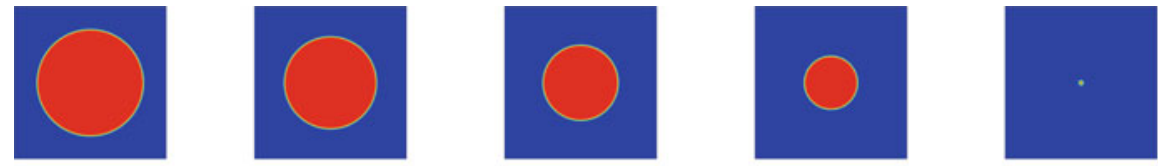

(a) $\mathrm{s}=1$
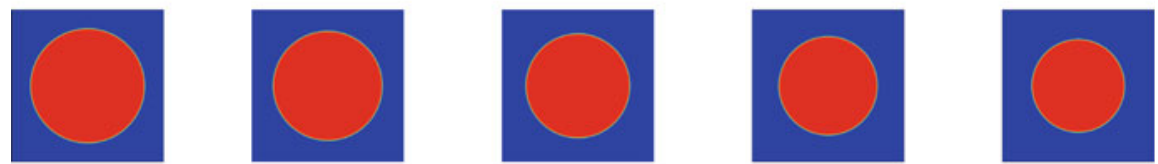

(b) $\mathrm{s}=0.9$
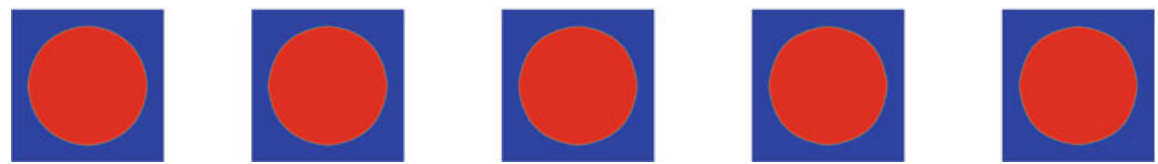

(c) $\mathrm{s}=0.8$

Fig. 4 Temporal evolution of a circular domain from left to right at times $t=$ $1000,2000,3000,4000,5000$, for fractional order $s=1(\mathbf{a}), 0.9(\mathbf{b}), 0.8(\mathbf{c})$, for the top, middle and bottom rows, respectively

Acknowledgements The work was supported by NSFC/ANR joint program 51661135011 PHASEFIELD. The second author has received financial support from the French State in the frame of the "Investments for the future" Programme Idex Bordeaux, reference ANR-10-IDEX03-02. The third author was also supported by NSFC grant 11971408, NNW2018-ZT4A06 project.

\section{References}

1. Allen, S.M., Cahn, J.W.: A microscopic theory for antiphase boundary motion and its application to antiphase domain coarsening. Acta Metall. 27(6), 1085-1095 (1979)

2. Shen, J., Yang, X.: Numerical approximations of Allen-Cahn and Cahn-Hilliard equations. Discrete Contin. Dynam. Syst. 28(4), 1669-1691 (2010)

3. Feng, X., Prohl, A.: Numerical analysis of the Allen-Cahn equation and approximation for mean curvature flows. Numer. Math. 94(1), 1669-1691 (2003)

4. Shen, J., Xu, J., Yang, J.: A new class of efficient and robust energy stable schemes for gradient flows (2017). arXiv:1710.01331 [math.NA]

5. Shen, J., Xu, J., Yang, J.: The scalar auxiliary variable (SAV) approach for gradient flows. J. Comput. Phys. 353, 407-416 (2018)

6. Lischke, A., Pang, G., Gulian, M., Song, F., Glusa, C., Zheng, X., Mao, Z., Cai, W., Meerschaert, M.M., Ainsworth, M., Karniadakis, G.E.: What is the fractional Laplacian? ArXiv:1801.09767V1 [MATH. NA] (29 Jan 2018)

7. Song, F., Xu, C., Karniadakis, G.E.: A fractional phase-field model for two-phase flows with tunable sharpness: algorithms and simulations. Comput. Methods Appl. Mech. Eng. 305, 376404 (2016)

8. Deville, M., Fischer, P.F., Mund, E.: High-order methods for incompressible fluid flow. Appl. Mech. Rev. 56(3), B43 (2002) 
9. Shen, J., Tang, T., Wang, L.-L.: Spectral Methods: Algorithms, Analysis and Applications, 1st edn., pp. 300-305. Springer, Berlin (2011)

10. Song, F., Xu, C., Karniadakis, G.E.: Computing fractional Laplacians on complex-geometry domains: algorithms and simulations. SIAM J. Sci. Comput. 39(4), A1320-A1344 (2017)

11. Chen, L.Q., Shen, J.: Applications of semi-implicit Fourier-spectral method to phase field equations. Comput. Phys. Commun. 108(2), 147-158 (1998)

Open Access This chapter is licensed under the terms of the Creative Commons Attribution 4.0 International License (http://creativecommons.org/licenses/by/4.0/), which permits use, sharing, adaptation, distribution and reproduction in any medium or format, as long as you give appropriate credit to the original author(s) and the source, provide a link to the Creative Commons licence and indicate if changes were made.

The images or other third party material in this chapter are included in the chapter's Creative Commons licence, unless indicated otherwise in a credit line to the material. If material is not included in the chapter's Creative Commons licence and your intended use is not permitted by statutory regulation or exceeds the permitted use, you will need to obtain permission directly from the copyright holder. 